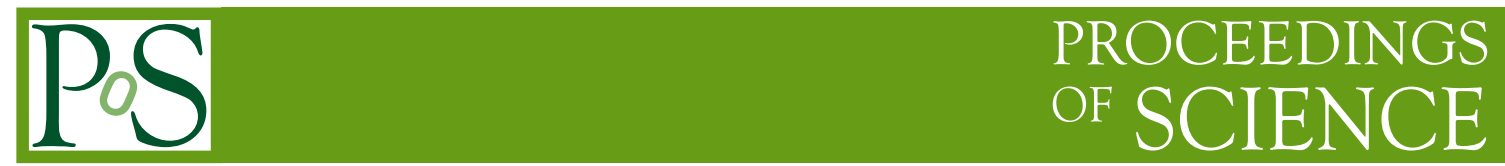

\title{
Mirror (a)symmetry far from stability
}

\author{
S. M. Lenzi* \\ Dipartimento di Fisica e Astronomia, Università di Padova and INFN, Padova, Italy \\ E-mail: lenziapd.infn.it \\ R. Lau \\ INFN Sezione di Padova, Padova, Italy \\ E-mail: lau@pd.infn.it
}

\begin{abstract}
Differences of excitation energy among analogue states in a broad mass range are calculated in the framework of the shell model by introducing isospin breaking interactions. Mirror and triplet energy differences along the $\mathrm{N}=\mathrm{Z}$ line from mass $A \sim 20$ to $A \sim 70$ have been systematically studied using the same method. It is shown that in all cases an additional term not directly associated to the electromagnetic interaction is needed to reproduce the experimental data.
\end{abstract}

X Latin American Symposium on Nuclear Physics and Applications (X LASNPA),

1-6 December 2013

Montevideo, Uruguay

${ }^{*}$ Speaker. 


\section{Introduction}

The study of differences in excitation energy between analogue states in isobaric multiplets allows to verify the validity of isospin symmetry and independence as a function of the angular momentum. These differences are of the order of tens of $\mathrm{keV}$ and can be well reproduced by state-of-the-art shell model calculations for nuclei in the $f_{7 / 2}$ shell [1,2]. Several nuclear structure properties can be deduced from these data, such as the alignment of nucleons along rotational bands, the evolution of the nuclear radius and the identification of pure single-particle excitations across two main shells. From the comparison with the available experimental data in nuclei of the $f_{7 / 2}$ shell, it is suggested that an additional isospin breaking term has to be considered. Two schematic terms consisting on only one matrix elements have been proposed by Zuker and collaborators [1]. In the case of the differences between excited states in mirror nuclei, the matrix element corresponds to two protons in the $f_{7 / 2}$ coupled to $J=2$, while for the energy differences of an isobaric triplet, the two protons are coupled to $J=0$. In both cases the strength of the matrix elements is $100 \mathrm{keV}$ and is deduced from the experimental data in mass $A=42$.

These theoretical studies have been carried out in parallel to the experimental developments that have allowed to follow the analogue states up to high spins due to the increase on the efficiency and resolution of gamma spectrometers. A compilation of these investigations are reported in the review article of ref. [2], where the same prescription was used for all nuclei in the $f_{7 / 2}$ shell, obtaining an excellent description of the data. In the last years the experimental studies have been extended from the nuclei in the $f_{7 / 2}$ shell to other mass regions due to the progress in the experimental techniques and the use of radioactive beams. From the theoretical side the research of the origin of the isospin breaking interaction is exploring different scenarios.

In this contribution, we present the results of an extension of these studies to nuclei in the $s d$, upper $p f$ shell and the $f p g$ space.

\section{Shell model calculations}

The mirror energy differences (MED) as a function of the nuclear spin $J$ are defined as:

$$
\operatorname{MED}(J)=E_{x}\left(J, T, T_{z}\right)-E_{x}\left(J, T,-T_{z}\right) .
$$

The triplet energy differences (TED), which refer to three isobaric nuclei with $T=1$, are defined as:

$$
\operatorname{TED}(J)=E_{x}\left(J, T=1, T_{z}=-1\right)+E_{x}\left(J, T=1, T_{z}=+1\right)-2 E_{x}\left(J, T=1, T_{z}=0\right),
$$

where $E_{x}$ are the excitation energies of the states referred to the ground state or to the lowest state of the same $\mathrm{T}$ in each nucleus and $T_{z}=(N-Z) / 2$.

To calculate these differences in the framework of the shell model, we follow the method described in refs. [1,2]. The terms that contribute to the energy differences can be divided in a monopole Coulomb, $V_{C m}$, a multipole Coulomb, $V_{C M}$, and an isospin breaking term $V_{B}$. MED and TED are therefore obtained by the following expressions:

$$
\operatorname{MED}(J)=\Delta_{M}<V_{C m}(J)>+\Delta_{M}<V_{C M}(J)>+\Delta_{M}<V_{B}(J)>
$$




$$
\operatorname{MED}(J)=\Delta_{T}<V_{C m}(J)>+\Delta_{T}<V_{C M}(J)>+\Delta_{T}<V_{B}(J)>
$$

where $\Delta_{M}$ and $\Delta_{T}$ indicate that the differences are obtained as in eq.(2.1) and (2.2), respectively.

Monopole Coulomb contributions are responsible for single particle shifts and bulk effects, while the multipole term takes into account the interaction between valence nucleons. The singleparticle energy corrections have two different origins. The energy of the proton orbits is affected proportionally to the square of the orbital momentum $l$ in the harmonic oscillator representation. In particular, the single-particle energy of protons with principal quantum number $N$, above the closed shell $Z_{C S}$ is modified by the following term [3]:

$$
E_{l l}=\frac{-4.5 Z_{C S}^{13 / 12}[2 l(l+1)-N(N+3)]}{A^{1 / 3}(N+3 / 2)}
$$

The relativistic electromagnetic spin-orbit interaction, that affects both the proton and neutron single-particle energy is $[4,5]$ :

$$
V_{l s}=\left(g_{s}-g_{l}\right) \frac{1}{2 m_{N}^{2} c^{2}}\left(\frac{1}{r} \frac{d V_{c}}{d r}\right) \vec{l} . \vec{s},
$$

where $m_{N}$ is the nucleon mass, and $g_{s}$ and $g_{l}$ are the giromagnetic factors of protons and neutrons.

There is an additional monopole effect due to the Coulomb interaction. It is normally called the radial term. This is due to changes of the nuclear radius as a function of the angular momentum. The radius of the nucleus depends on the orbitals that are occupied and they may change with $J$. The radial term can be calculated in the shell model framework as discussed in ref.[1] from the equation below:

$$
V_{C m}=n \alpha_{r}\left(\frac{m_{\pi}(\text { g.s. })+m_{v}(\text { g.s. })}{2}-\frac{m_{\pi}(J)+m_{v}(J)}{2}\right)
$$

where $n / 2=\left|T_{z}\right|, m_{\pi}, m_{v}$ are the proton and neutron occupation numbers. $\alpha_{r}$ is a parameter and depends on the orbits.

In refs. [1] it was shown that the effect of the isospin breaking interaction $V_{B}$ in both MED and TED can be as large at the Coulomb terms. They were deduced from extracting the Coulomb contribution to the MED and TED in mass $A=42$. The extrapolation to other shells is not straightforward. In mass $\mathrm{A}=42$, the wavefuntions can be considered of pure $f_{7 / 2}$ nature while this is not the case for other shells. In this work we explore the possibility of using a similar prescription as that of the $f_{7 / 2}$, trying to find the relevant matrix elements (always limited to only one term) with a strength that allows a good description of all the available data.

\section{Results}

We calculate the mirror energy differences and the triple energy differences of nuclei from mass range $\mathrm{A} \sim 20$ up to $\mathrm{A} \sim 70$ where experimental values are available. The calculations were done by using the shell-model code ANTOINE $[6,7]$ with different effective nuclear interactions. For the sd shell, the USD interaction [8,9] has been used while the KB3G [10] is used for nuclei in the $f_{7 / 2}$ shell. For $\mathrm{A}=57$ to $\mathrm{A}=61$, the GXPF1A [11] interaction has been adopted. Above $\mathrm{A}=66$ the valence space includes $p_{3 / 2}, f_{5 / 2}, p_{1 / 2}$ and $g_{9 / 2}$ orbitals. For this model space the JUN45 interaction [12] has been used. 
In this section, we show some few illustrative examples of the results obtained for MED and TED of nuclei in the sd shell and upper fp shell. The result of MED and TED of the $f_{7 / 2}$ shell can be found in [2].

\subsection{MED and TED in the sd shell}

The calculations of the MED and TED in the $s d$ shell are in very good overall agreement with the available data. We report in Fig. 1 the results for mass $\mathrm{A}=22$, together with the single contributions. The radial term in this case is due to the changes in occupation number of the $s_{1 / 2}$ orbit with strength $\alpha=100 \mathrm{keV}$. For the $V_{B}$ term we consider a matrix element only with two protons in the $d_{5 / 2}$ coupled to $J=2$ with a strength of $100 \mathrm{keV}$, similar to that used in the $f_{7 / 2}$ shell. Following the prescription of ref. [1,2], for the TED, the $V_{B}$ term corresponds to a single matrix element with two protons in the $d_{5 / 2}$ coupled to $J=0$ with a strength of $100 \mathrm{keV}$. In Fig. 1 the MED is not so well described for $A=22$, while the TED are in very good agreement. The rest of the masses are much better described for the MED, but we have chosen $\mathrm{A}=22$ because there are data for the TED. The contribution of the $V_{B}$ term in the MED for $\mathrm{A}=22$ is not very important, but it is necessary in other masses. Its contribution to the TED can be clearly deduced from the figure.

MED

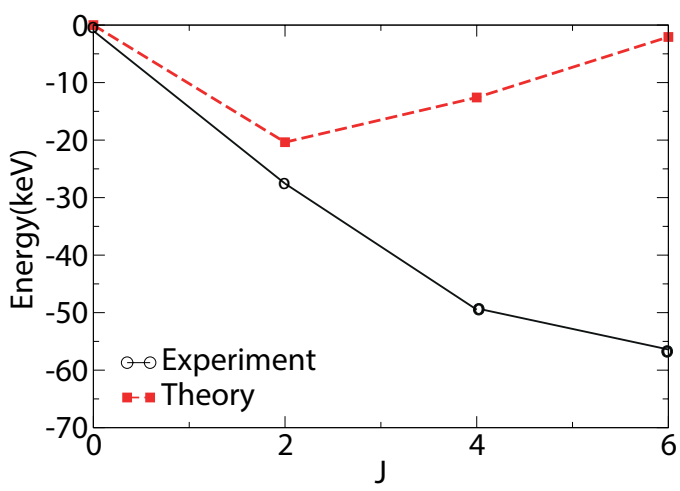

MED different components

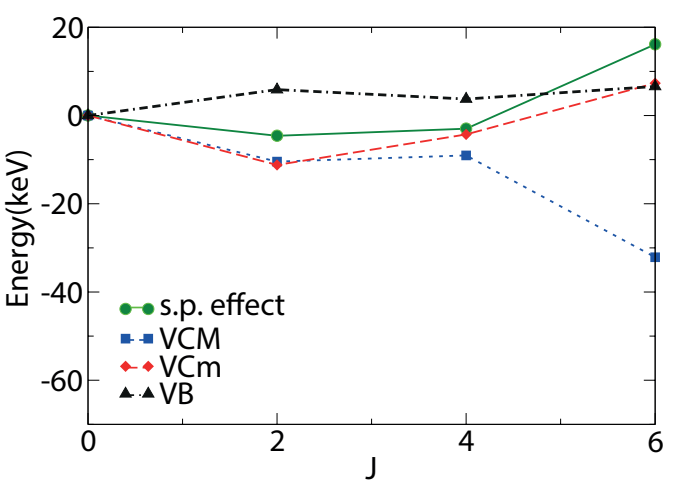

TED

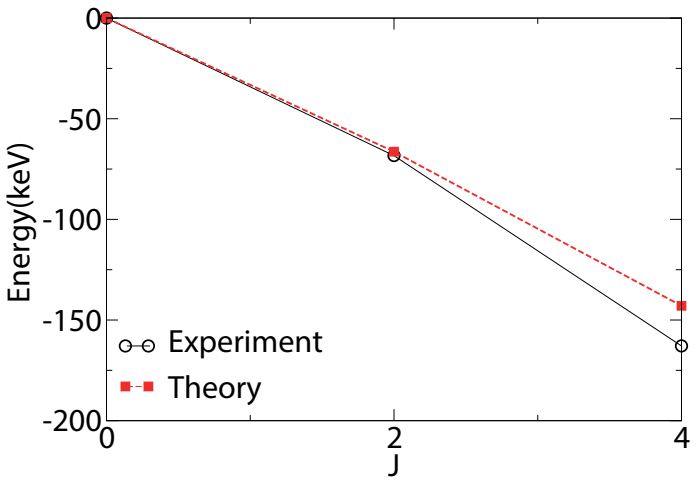

TED different components

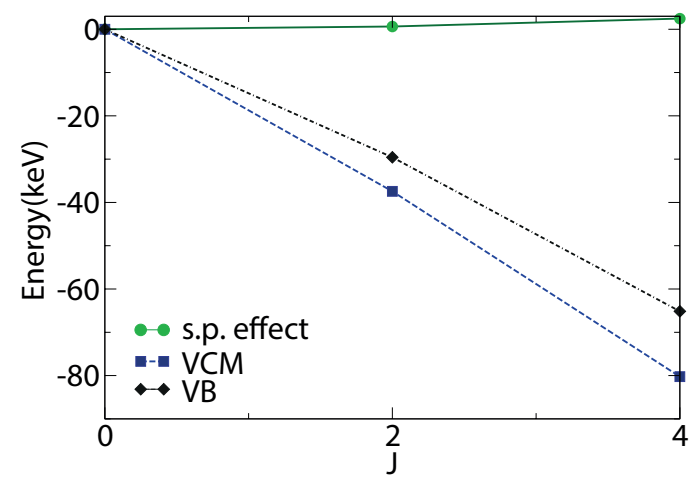

Figure 1: MED (left panels) and TED (right panels) for mass $A=22$. The lower plots show the contribution of the different components of MED and TED. 


\subsection{MED and TED of masses in the mass range $A=57$ to $A=61$}

The calculations of the MED and TED in the upper $f p$ shell are in overall agreement with the available data. We report in Fig. 2 the results for mass $A=58$, together with the single contributions. As in ref. [1,2], the radial term is due to changes in the occupation number of the $p_{3 / 2}$ orbit with strength $\alpha=200 \mathrm{keV}$. For the $V_{B}$ term we consider a matrix element with two protons in the $p_{3 / 2}$ coupled to $J=0$ with a strength of $-100 \mathrm{keV}$. For the TED, the $V_{B}$ term corresponds to a single matrix element with two protons in the $p_{3 / 2}$ coupled to $J=0$ with a strength of $100 \mathrm{keV}$. Both MED and TED are in very good agreement with the data. The contribution of the $V_{B}$ term is clearly relevant.

MED

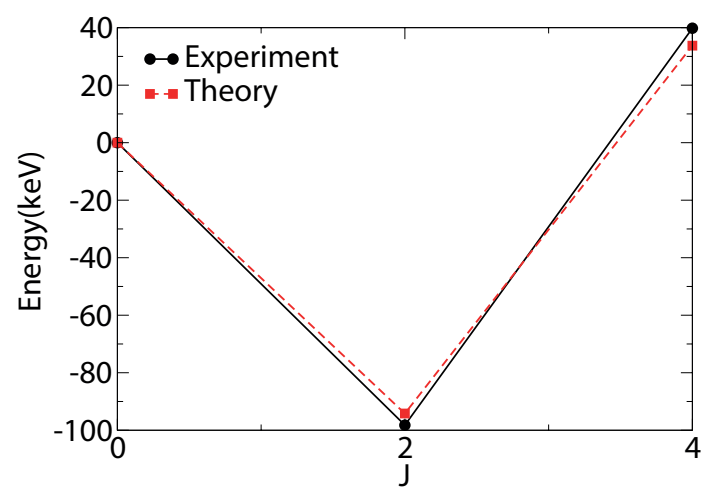

MED different components

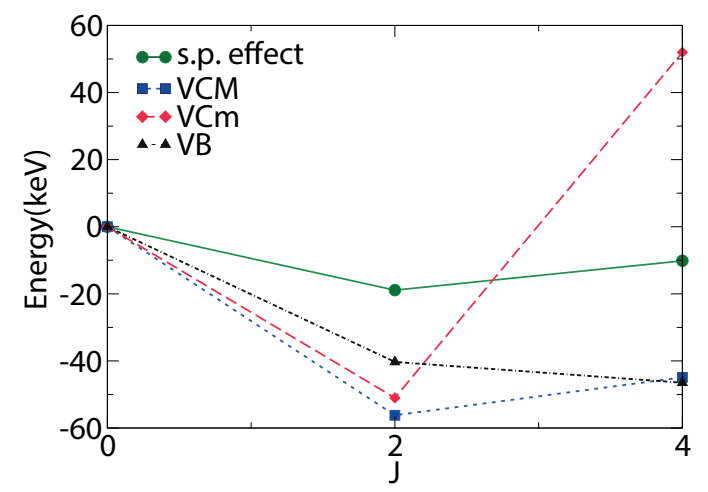

TED

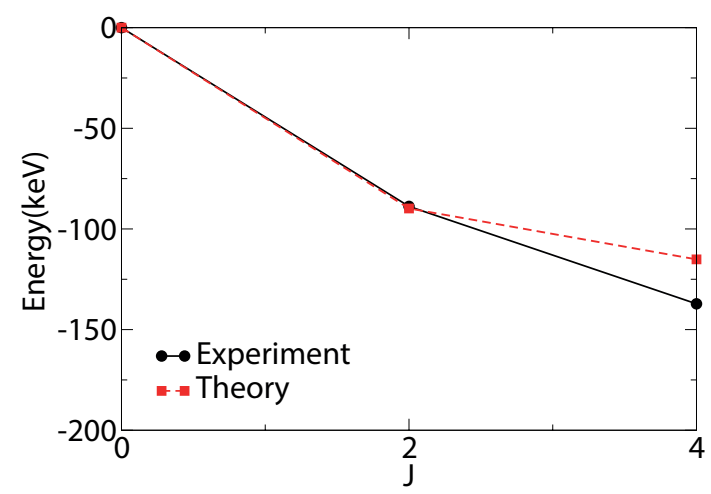

TED Different Component

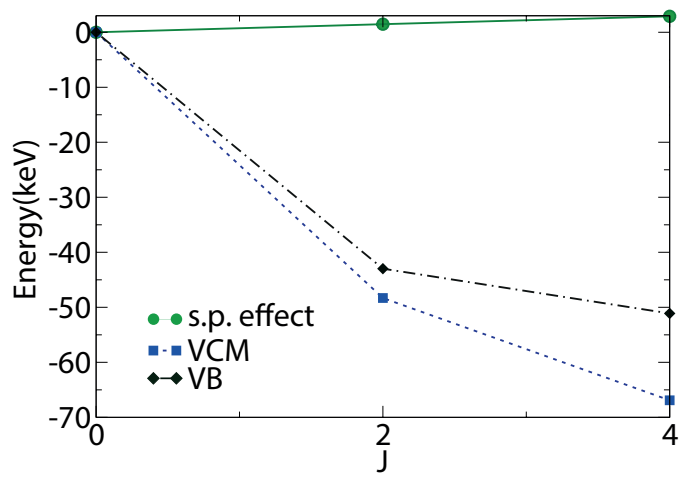

Figure 2: MED (left panels) and TED (right panels) for mass A=58. The lower plots show the contribution of the different components of MED and TED.

\subsection{MED and TED in the mass range $A=66$ to $A=70$}

MED and TED in the fpg shell are also in very good overall agreement with the available data. We report in Fig. 3 the results for mass $A=66$, together with the single contributions. Here the radial term is due to changes in the occupation number of both the $p_{3 / 2}$ and the $g_{9 / 2}$ orbit with strengths $\alpha=200 \mathrm{keV}$ and $-300 \mathrm{keV}$, respectively. The difference in the sign is due to the fact that the increasing of the occupation of the $g_{9 / 2}$ orbital make the Coulomb repulsion to decrease. 

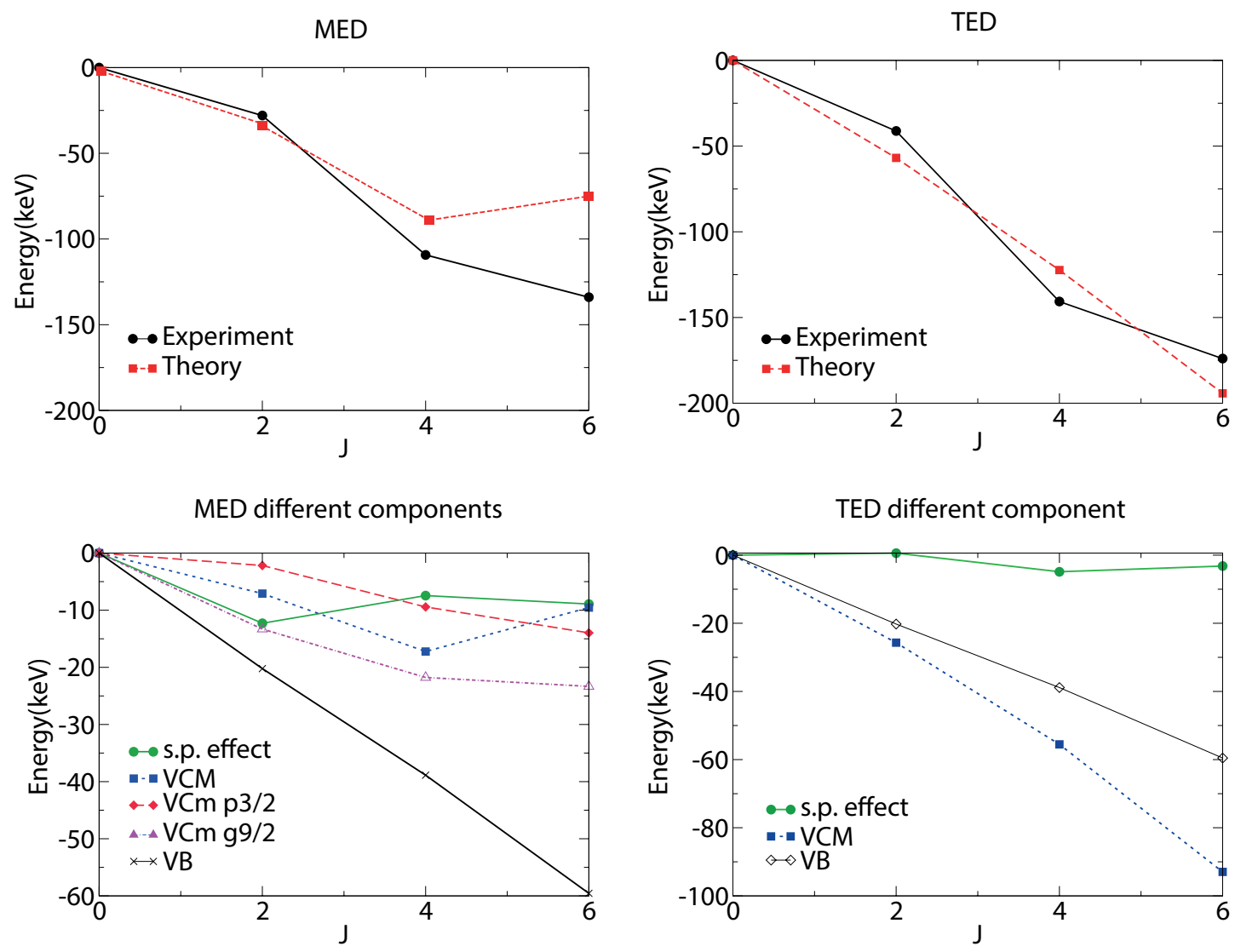

Figure 3: MED (left panels) and TED (right panels) for mass $A=66$. The lower plots show the contribution of the different components of MED and TED.

For the $V_{B}$ term we consider a matrix element with two protons in the $f_{5 / 2}$ coupled to $J=0$ with strength of $300 \mathrm{keV}$. This is last term is not on the line of what has been assumed in the other mass regions, more data is needed here to arrive to a final conclusion. In any case, we have chosen to take into account all terms in the calculation of the MED at odd with other publications where a not all terms that contribute to the MED have been taken into account [13]. For the TED, the $V_{B}$ term corresponds to a single matrix element with two protons in the $f_{5 / 2}$ coupled to $J=0$ with strength of $100 \mathrm{keV}$.

\section{Conclusions}

In the present work we have performed a systematic study of the MED and TED in different mass regions that cover the $s d$ shell up to the $f p g$ shell. The theoretical calculations have explored with the same prescription the different mass regions. In all cases so far investigated the need of an additional isospin breaking term $V_{B}$ is needed to reproduce the data. Here we present some significant examples but it is important to note that the same parameterization reproduces all cases for which data are available. A more detailed presentation of the results, that include new data is in preparation. 


\section{References}

[1] A. P. Zuker, S. M. Lenzi, G. Martinez-Pinedo and A. Poves, Isobaric multiplet yrast energies and isospin nonconserving forces, Phys. Rev. Lett. 89, (2002) 142502.

[2] M. Bentley and S. M. Lenzi, Coulomb energy differences between high-spin states in isobaric multiplets, Prog in Part. and Nucl. Phys. 59, (2007) 497.

[3] J. Duflo and A. P. Zuker, Mirror displacement energies and neutron skin, Phys. Rev. C 66, (2002) 051394(R).

[4] J. A. Nolen and J. P. Schiffer, Coulomb energies, Annu. Rev. Nucl. Sci. 19, (1969) 471.

[5] J. Ekman et al., Unusual isospin-breaking and isospin-mixing effects in the A=35 mirror nuclei, Phys. Rev. Lett. 92, (2004) 1322502.

[6] E. Caurier, shell model code ANTOINE, IRES, Strasbourg, 1989-2002.

[7] E. Caurier and F. Nowacki, Present status of shell model techniques, Acta. Phys. Vol. 30, (1999) 705.

[8] B. H. Wildenthal, Empirical strengths of spin operators in nuclei, Prog in Part. and Nucl. Phys. 11, (1984) 5 .

[9] B. A. Brown, B. H. Wildenthal, Status of the nuclear shell model, Annu. Rev. Nucl. Part. Sci. 38, (1988) 29.

[10] A. Poves, J. Sánchez-Solano, E. Caurier and F. Nowacki, Shell model study of the isobaric chains $A=50, A=51$ and $A=52$, Nuclear Phys. A 694, (2001) 157.

[11] M. Honma, T. Otsuka, B. A. Brown and T. Mizusaki, Effective interaction for pf-shell nuclei, Phys. Rev. C 65, (2002) 061301.

[12] M. Honma, T. Otsuka, T. Mizusaki and M. Hjorth-Jensen, New effective interaction for $f_{5} p g_{9}$ shell nuclei Phys. Rev. C 80, (2009) 064323.

[13] K. Kaneko, S. Tazaki, T. Mizusaki, Y. Sun, M. Hasegawa and G. de Angelis, Isospin symmetry breaking at high spins in the mirror pair ${ }^{67}$ Se and ${ }^{67}$ As, Phys. Rev. C 82, (2010) 061301. 